\title{
Sostenibilidad del volumen de cosecha calculado con el Método Mexicano de Ordenación de Montes
}

\author{
Juan Manuel Torres Rojo
}

\begin{abstract}
RESUMEN
Se presenta un análisis del efecto del volumen de corta estimado con el Método Mexicano de Ordenación de Montes (MMOM) sobre la sustentabilidad del bosque. En el análisis se postula que la proyección del crecimiento de un bosque debe seguir un comportamiento logístico y no un comportamiento exponencial como lo supone el MMOM. Si la proyección del crecimiento sigue un comportamiento logístico entonces es posible regular la corta en el largo plazo e identificar, en el corto plazo, criterios para evaluar la sostenibilidad de un volumen de corta y la sustentabilidad de la masa remanente. De igual forma, es posible identificar un volumen de cosecha económicamente óptimo. Por el contrario, el supuesto de crecimiento exponencial no permite regular la corta en el largo plazo ni tampoco identificar indicadores de sustentabilidad. Adicionalmente, no permite estimar un volumen de cosecha económicamente óptimo. Los resultados muestran que el MMOM debe ser modificado en su supuesto de crecimiento exponencial y que se debe enfatizar el manejo de estructuras residuales a fin de lograr la sustentabilidad de los bosques mexicanos manejados bajo sistema irregular.
\end{abstract}

PALABRAS CLAVE:

Regulación por volumen, cosecha, manejo irregular, sustentabilidad.

\begin{abstract}
An analysis considering the effect of harvest volume estimated through the Mexican Method of Forest Regulation (MMFR) on the sustainability of forest is presented. The analysis postulates that growth projection of a forest population must follow a logistic behavior, instead of an exponential behavior as assumed by the MMFR. When growth projection follows a logistic behavior, then harvest regulation is possible in the long run and it is easy to identify criteria to evaluate sustained yield and the sustainability of the residual forest in the short run. In addition, it is possible to identify economically optimal harvest volumes. On the other hand, the assumption of exponential growth does not allow neither harvest regulation nor sustainability criteria identification. In addition it is not possible to estimate an economically optimal harvest volume. Results show that the assumption of exponential growth from the MMFR must be modified and the management of residual forest structures must be emphasized in order to achieve sustainability on the Mexican forests managed under an irregular system.
\end{abstract}

KEY WORDS:

Volume control, harvest, irregular management, sustainability

1 Centro de Investigación y Docencias Económicas. Carr. México-Toluca No. 3655. Col. Lomas de Santa Fe. 01210 México, D.F. c.e.: torresrj@dis1.cide.mx.

Manuscrito recibido el 18 de noviembre de 1999. Aceptado el 3 de marzo del 2000. 


\section{INTRODUCCIÓN}

En los últimos años ha crecido el interés de la sociedad por conocer mejor la condición en la que se encuentran los bosques tanto locales como a nivel mundial. Este cambio en gustos y preferencias ha llevado a cuestionamientos acerca de la forma en que se administran los bosques y selvas del planeta. Tales cuestionamientos han derivado temas de vital importancia sobre la condición de los bosques, entre los que se incluyen la deforestación y fragmentación forestal, la reducción en la productividad forestal, la pérdida de la biodiversidad y la marginación y debilitamiento paulatino de las comunidades dependientes del bosque; todos ellos temas que involucran no sólo aspectos técnicos de la administración de los bosques sino aspectos económicos y sociales. Ante este panorama, los estudiosos sobre la administración de bosques y selvas han desarrollado una nueva filosofía de administración de éstos denominada "Manejo Forestal Sustentable" (MFS). Esta filosofía ha emergido como una nueva estructura conceptual para describir un manejo forestal que asegure la salud y productividad del bosque en el largo plazo y que a la vez proporcione un flujo continuo de beneficios sociales y económicos para toda la sociedad y en particular para las comunidades locales. El concepto es muy amplio, y si bien es cierto que es complicado entenderlo en términos conceptuales resulta mucho más complicado tratar de llevarlo a la práctica. Es por ello que en la actualidad existen muchos esfuerzos a nivel mundial para intentar traducir este amplio concepto en principios, criterios e indicadores que reflejen un medio para evaluar y monitorear el progreso de las actividades de manejo forestal hacia un MFS. El presente trabajo se puede considerar parte de este esfuerzo, ya que busca identificar indicadores de sostenibilidad de la cosecha calculada a través del Método
Mexicano de Ordenación de Montes (MMOM).

El MMOM es un procedimiento para el cálculo de la cosecha de bosques irregulares de coníferas, ya sea puras o mezcladas con latifoliadas (Rodríguez, 1958). El Método tuvo su origen en los lineamientos oficiales dictados en octubre de 1944 para sujetar la formulación de proyectos de ordenación en bosques de zonas templado frías del país. Las bases teóricas del MMOM son ampliamente expuestas por Mendoza y Rodríguez (1959) y extendidas en DGAF (1984b). El MMOM es tan sólo un procedimiento para calcular el volumen de cosecha de bosques irregulares, mismo que supone que un rodal crece a lo largo de un periodo a una tasa constante sobre el volumen residual. De aquí que el volumen de cosecha se calcula con tan sólo estimar el volumen de crecimiento sobre tal periodo. Hacia, 1978 el procedimiento fue severamente criticado por degenerar en un procedimiento totalmente selectivo de arbolado maduro con un diámetro mínimo, por lo que se adecuó (DGAF, 1984a) para que la corta se distribuyera en todas las categorías diamétricas, procurando dejar una estructura residual balanceada, lo que se denominó Método Mexicano de Ordenación de Bosques Irregulares (MMOBI). Sin embargo, el cálculo del volumen de cosecha siguió las mismas bases del MMOM sin incorporar conceptos de optimización de estructuras residuales (DGAF, 1984a) u otro tipo de procedimiento expedito para asegurar un manejo de estructuras residuales (Guldin, 1991; Long, 1998).

En la actualidad se sabe que el mantener una estructura balanceada puede sostener un nivel de cosecha indefinidamente (Leak y Filip, 1977; Reynolds et al., 1984; Seymour y Kenefic, 1998) además de conservar el bosque, por lo que se dice que la estrategia es sustentable (O'Hara, 1998). Sin embargo, también se sabe que otro tipo de 
estructuras también pueden mantener un nivel de cosecha y conservar el bosque, sin la restricción de que sean balanceadas (Adams y Ek, 1974; Haight et al., 1985; O'Hara, 1996; Seymour y Kenefic, 1998). De aquí que la restricción de mantener una estructura balanceada en el manejo moderno de bosques irregulares no es relevante. Incluso en algunas regiones se ha comprobado que la política de mantener una estructura balanceada ha originado que los administradores del bosque pierdan de vista aspectos de mayor relevancia para la sustentabilidad del bosque, como aspectos estructurales más significativos, entre los que se pueden señalar: distribución del follaje, estratificación de la masa o la misma ocupación de copas; variables estrechamente relacionadas con la sustentabilidad del bosque y la producción de múltiples bienes y servicios (Seymour y Kenefic, 1998; O’Hara, 1998).

Lo anterior pone de manifiesto que el manejo forestal con fines maderables a través de un régimen irregular no requiere de conservar estructuras balanceadas, sino más bien debe enfatizar un nivel de población (densidad) que asegure las sostenibilidad de la cosecha y el manejo de variables estructurales en las existencias residuales que permitan mantener la salud, productividad y producción de múltiples bienes y servicios. Con este marco de referencia resulta relevante analizar el procedimiento de cálculo de cosecha con el MMOM, dado que si el nivel de cosecha que se obtiene es sostenible, entonces con sólo enfatizar algunas prácticas de manejo de la estructura residual se puede catalogar como un procedimiento sustentable.

El presente artículo trata de identificar las condiciones (parámetros) bajo las cuales el volumen de cosecha estimado a partir del Método Mexicano de Ordenación de Montes (MMOM) puede asegurar la sustentabilidad del recurso bosque. El tema reviste una importancia singular dado que en la actualidad todavía un alto porcentaje de los bosques del país son administrados bajo el MMOM. El artículo se organiza de la siguiente forma. En la siguiente sección se expone brevemente el MMOM y se establecen algunas deficiencias básicas. Así mismo, se indica un modelo que puede ayudar a mitigar tales deficiencias, mismo que se utiliza para realizar un análisis del volumen de cosecha calculado con el MMOM. Posteriormente se resumen algunos resultados importantes del modelo propuesto y del MMOM y se derivan algunos indicadores para evaluar la sostenibilidad del nivel de cosecha calculado con el MMOM. Finalmente se presentan algunas conclusiones.

\section{MATERIALES Y METODOS}

\section{EI MMOM y sus supuestos}

Como es sabido el MMOM basa el cálculo del volumen de cosecha en la fórmula del interés compuesto. Tal fórmula establece que el volumen que se puede obtener al año $t\left(V_{t}\right)$ no es más que el volumen inicial $\left(\mathrm{V}_{0}\right)$ más el volumen producto del crecimiento de la masa forestal, mismo que se presume crece a una tasa constante y compuesta ( $r$ ) sobre el volumen en pie y durante el periodo $0 \rightarrow$ $t$. La estimación es como sigue:

$$
\mathrm{V}_{\mathrm{t}}=\mathrm{V}_{0}(1+\mathrm{r})^{\mathrm{t}}
$$

Donde:

$$
\begin{aligned}
\mathrm{V}_{\mathrm{t}}= & \text { existencias volumétricas actuales } \\
\mathrm{V}_{0}= & \text { volumen remanente una vez } \\
& \text { realizada la cosecha } \\
\mathrm{t}= & \text { periodo de crecimiento o ciclo de } \\
& \text { corta } \\
\mathrm{r}= & \text { tasa de crecimiento, teóricamente } \\
& \text { calculada como }
\end{aligned}
$$

$$
r=\frac{V}{V}=\frac{\frac{d V}{d t}}{V}
$$


que en la práctica corresponde a un promedio del crecimiento anual en un periodo anterior. De (2) el crecimiento se relaciona con el volumen a través de la expresión:

$$
g(V)=V=\frac{d V}{d t}=r V
$$

relación que implica un crecimiento proporcional al volumen. Bajo este enfoque resulta relativamente sencillo estimar el volumen de cosecha sí se conoce la tasa de crecimiento $(r)$ y el periodo de tiempo (t) de recuperación de la masa (ciclo de corta). Así el MMOM estima el volumen de cosecha $(\mathrm{H})$ por:

$$
\left.H=V_{t}-V_{0}=V_{0} \mid(1+r)^{t}-1\right]
$$

o en términos continuos:

$$
H=V_{t}-V_{0}=V_{0}\left[e^{r t}-1\right]
$$

donde $V_{0}$ nuevamente representa el volumen residual de la masa que teóricamente será capaz de recuperar las existencias reales $\left(V_{t}\right)$ que se tienen originalmente.

El modelo del MMOM para el cálculo de la cosecha es obviamente muy simple y eficiente. Sin embargo la estimación del volumen de cosecha en (4) tiene varios supuestos implícitos que es necesario destacar, entre los cuales sobresalen:
a) Las existencias actuales $\left(V_{t}\right)$ representan el inventario óptimo que se debe recuperar y conservar.
b) La tasa de crecimiento ( $r$ ) en el periodo $0 \rightarrow$ t es constante en todo el periodo.
c) La tasa de crecimiento ( $r$ ) se puede lograr en el periodo $t \rightarrow 2 t$, sin depender de la estructura de tamaños del bosque remanente.

d) La incorporación y mortalidad no intervienen en la determinación del crecimiento.

El primer supuesto puede ser bastante controversial. Si la masa original es una masa natural que no ha sido cosechada, entonces muy probablemente las existencias volumétricas son muy cercanas a la capacidad de carga del sitio. En tal caso y considerando cualquier población con recursos limitantes, la tasa de crecimiento es muy baja y obviamente no convendría, desde el punto de vista de administración del bosque, conservar una masa con tasas de crecimiento tan bajas. Por el contrario, dado que una masa natural no puede tener su equilibrio cuando la tasa de crecimiento es máxima (Clark, 1990), entonces es muy probable que la masa natural no presenta la mayor tasa de crecimiento. Evidentemente con fines de explotación maderable el inventario óptimo a conservar será aquel que una vez terminado el ciclo de corta proporcione el máximo crecimiento maderable. Esto significa que el inventario a conservar depende del ciclo de corta, de la tasa de crecimiento y de las existencias residuales.

El segundo supuesto señala que la tasa de crecimiento es constante a lo largo del periodo (ciclo de corta) y acumulable con la densidad, esto es, sigue un comportamiento exponencial como el mostrado en la figura 1.

El supuesto es bastante fuerte dado que toda población tiene una tasa de crecimiento dinámica. Incluso es común que en reportes de evaluación sobre el MMOM se señale el hecho de que en algunos sitios el procedimiento brinda resultados positivos, entendiendo por ello que las existencias crecieron a un nivel igual o superior al esperado, mientras que en otras zonas los resultados son negativos. En algunos documentos incluso se señala que eso es el resultado de que 


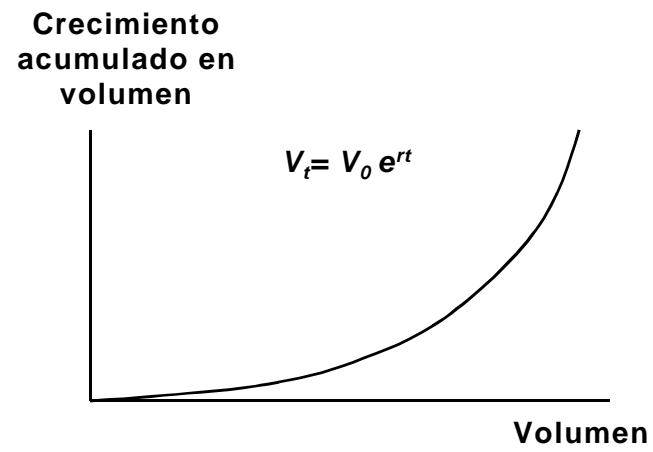

Figura 1. Crecimiento acumulado suponido por el MMOM.

en unos sitios el arbolado crece con la fórmula del interés compuesto y en otros con la del interés simple (Mendoza y Rodríguez, 1959). Varios autores han estudiado la relación entre el volumen total de un rodal (como medida aproximada del grado de competencia) y el crecimiento en volumen para diferentes especies y en diferentes condiciones de crecimiento (Lagsaeter, 1941; Mar-Moller, 1960; Davis, 1956; Assmann, 1970; Smith, 1986; Oliver y Larson, 1990).

En todos los casos se coincide en que la tasa de crecimiento de una población tiene rendimientos marginales decrecientes, esto es, es muy acelerada cuando la población es poco densa, logra un máximo y cae paulatinamente a medida que la población crece. Así por ejemplo, si el número y tamaño de los individuos en la población es muy cercano a la capacidad de carga, entonces la tasa de crecimiento será muy baja y aproximada a cero. Por el contrario, si la densidad de la población es menor, entonces la tasa de crecimiento será mayor y obviamente existirá un rango de densidades sobre el cual existe una tasa máxima de crecimiento poblacional. Esta relación se presenta tanto en rodales manejados como aquellos no manejados y frecuentemente es referida como la relación de Lagsaeter (Oliver y Larson, 1990). La relación es obviamente variable con respecto a la especie, sitio y distribución inicial de tamaños, sin embargo se ha comprobado que tiene una forma similar a la presentada en la figura 2 para aquellos rodales espaciados irregularmente con alta diferenciación en tasas de crecimiento, esto es, rodales irregulares (Oliver y Larson, 1990).

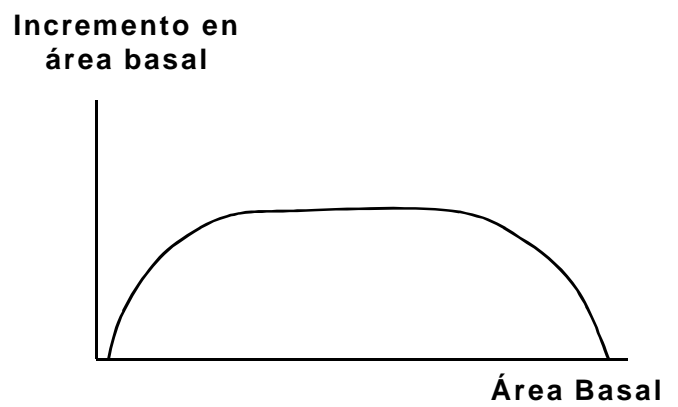

Figura 2. Relación de Langsaeter.

Sin duda este supuesto es el que mayor impacto tiene en el cálculo del volumen de cosecha, ya que mientras más largo sea el ciclo de corta se supone un periodo de tiempo más largo de crecimiento exponencial, mismo que una masa natural no puede sostener. Ello implica que mientras más largo es el ciclo de corta es más probable que el bosque no recupere las existencias que supuestamente debe tener.

El tercer supuesto es igualmente fuerte en el sentido de que se supone que dado un volumen residual la tasa de crecimiento de la población es la misma independientemente de la distribución de tamaños. Evidentemente el supuesto es muy restrictivo ya que el crecimiento poblacional es la suma de los crecimientos individuales, mismos que dependen de la estructura de tamaños y la proporción de especies.

El cuarto supuesto parece muy restrictivo pero podría no representar 
mayor problema si la tasa de crecimiento refleja el componente neto, esto es, existencias + incorporación - mortalidad. Existen sin lugar a dudas, otras críticas al MMOM que han sido ampliamente debatidas, tal es el caso de la ausencia de una estrategia ordenatoria o regulatoria de la cosecha, la ausencia de criterios de calidad de sitio 0 densidad en la determinación de la cosecha, o bien la falta de criterios claros en la aplicación de la cosecha. Sin embargo, estas críticas serán abordadas más adelante en la exposición. Por lo pronto la discusión se enfocará a los cuatro supuestos anteriores.

\section{Análisis del MMOM.}

Los supuestos (b), (c) y (d) podrían no ser limitantes si se supone que el comportamiento del crecimiento $(g(V))$ es variable con respecto a la densidad y constituye una tasa de crecimiento neto. Adicionalmente, si se supone que la variación en el crecimiento debido a diferencias en las estructuras residuales se puede incorporar suponiendo una variación en el volumen residual $\left(\mathrm{V}_{0}\right)$ dentro de un pequeño intervalo, entonces el supuesto (c) se hace totalmente irrelevante ${ }^{1}$. Por su parte el supuesto (a) está relacionado con el nivel más apropiado de densidad que debe tener la masa, mismo que constituye un problema de manejo de densidad, no abordado por el MMOM.

Con el fin de analizar el efecto del nivel de cosecha del MMOM conjetúrese dos formas de comportamiento del crecimiento de la masa, una con una tasa de crecimiento variable que tiene la forma de una función logística; esto es, una tasa que incrementa con rendimientos marginales decrecientes cuando la población tiene un volumen pequeño hasta lograr un valor máximo, a partir del cual la tasa de crecimiento tiende a disminuir

1 El efecto se puede modelar haciendo que $r$ sea una función de la estructura de la masa. Sin embargo, ello requiere una medida de la estructura y conocer la respuesta de $r$ a diferentes estructuras. conforme la población comienza a saturar el sitio y lograr una tasa igual a cero cuando se alcanza la capacidad de carga del sitio. Mientras que la otra es una masa que crece a una tasa constante, tal y como lo señala el MMOM. La figura 3 muestra la tendencia de ambas trayectorias donde $\mathrm{g}_{\mathrm{M}}$ identifica la trayectoria del crecimiento suponido con el MMOM y $g_{L}$ supone una función logística de crecimiento, misma que es similar (al menos en forma) a las tendencias tradicionales de crecimiento (Lagsaeter, 1941; Klepac, 1976; Oliver y Larson, 1990) supuestas incluso para el tipo de masa al que se aplica el MMOM (Rodríguez y Rodríguez, 1966).

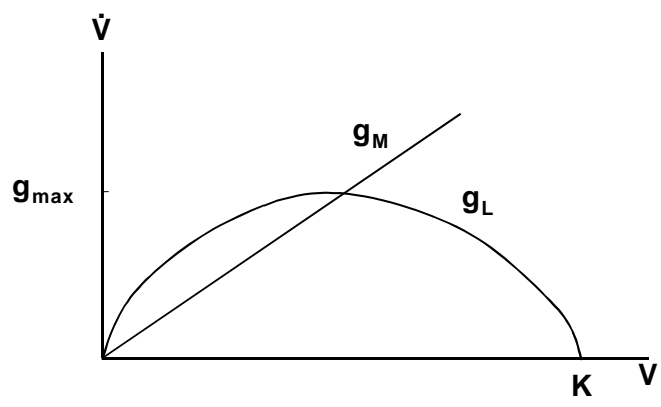

Figura 3. Curva de crecimiento logística vs crecimiento exponencial.

La tasa de crecimiento logístico se expresa algebraicamente como:

$$
g(V)=\frac{\dot{V}}{V}=\frac{\frac{d V}{d t}}{V}=r\left(1-\frac{V}{K}\right)
$$

Donde:

$\mathrm{K}$ = capacidad de carga del sitio (máximo volumen maderable en el sitio).

$\mathrm{V}=$ volumen de la masa al inicio del periodo de crecimiento.

$r$ = tasa de crecimiento exponencial (máximo).

$\mathrm{t}=$ tiempo 
Con base en este comportamiento, el crecimiento en volumen (V) de la población tiene la siguiente forma:

$$
\mathrm{V}=\mathrm{rV}\left(1-\frac{\mathrm{V}}{\mathrm{K}}\right)
$$

Resolviendo la ecuación diferencial se obtiene el siguiente resultado para estimar volumen en el tiempo $t\left(\mathrm{~V}_{\mathrm{t}}:\right)$

$$
V_{t}=\frac{K}{1+c e^{-r t}}
$$

Donde:

$$
\mathrm{C}=\frac{\mathrm{K}-\mathrm{V}_{0}}{\mathrm{~V}_{0}}
$$

de esta forma si se supone que el crecimiento real de la población tiene una tendencia logística, entonces el volumen de corta en un periodo t se puede expresar como:

$$
H=V_{t}-V_{0}=V_{0}\left[\frac{K}{V_{0}+\left(K-V_{0}\right) e^{-r t}}-1\right]
$$

volumen de corta que siempre será menor que aquel calculado por el MMOM (4) dada una misma $r$. Observe que si se supone que el volumen residual $\left(V_{0}\right)$ no es constante de ciclo de corta a ciclo de corta, entonces de acuerdo con (6) existe espacio para suponer crecimientos variables dado un mismo ciclo de corta y dado un volumen residual igualmente variable.

Para analizar el efecto del volumen de cosecha calculado con el MMOM sobre la sustentabilidad del bosque, a continuación se muestran cuatro estrategias de cosecha en las cuales se comparan los volúmenes de cosecha estimados con el MMOM con relación a un comportamiento de crecimiento más real como aquel definido por la función logística de crecimiento. a) Suponga que la cosecha en el periodo 0 - $\mathrm{t}\left(\mathrm{H}_{0}\right)$ es mayor al máximo crecimiento posible del bosque en el periodo t, esto es

$$
\mathrm{H}_{0}>\mathrm{t} \operatorname{máx}_{\mathrm{V}}\{\mathrm{g}(\mathrm{V})\}=\operatorname{tg}_{\text {máx }}=\mathrm{t} \frac{\mathrm{rK}}{4}
$$

donde $g_{\text {máx }}$ identifica el máximo nivel de crecimiento anual suponiendo un crecimiento logístico.

Si se considera que la masa sigue el crecimiento logístico resulta evidente que el volumen de cosecha en el periodo $t$ (p.e.: $\mathrm{H}_{0}=\mathrm{t} \mathrm{g}_{\text {máx }}$ ) es demasiado alto (Fig. 4) y que la masa requerirá de un periodo más largo que t para poder recuperarse. Resultado evidente de esta estrategia de cosecha es que si se continua con el mismo nivel de cosecha $\left(\mathrm{H}_{0}\right)$ y la misma longitud del ciclo de corta $(\mathrm{t})$, entonces el bosque perderá tanta densidad que se liquidará completamente. De aquí que no resulta sostenible este nivel de cosecha. El caso extremo es que $H_{0}=V_{t}$, lo cual significa una liquidación total de la masa en un sólo corte.

Si por el contrario se considera que la masa sigue un patrón de crecimiento exponencial como el definido por el MMOM, entonces resulta evidente que siempre (para cualquier $r>0$ ) se podrá recuperar el volumen de cosecha independientemente de la magnitud de $r$. Así lo muestran los puntos A, B y C de la figura 4, que son puntos en los cuales el volumen de cosecha $\mathrm{H}_{0}$ es igual al volumen de crecimiento $\mathrm{V}$ para tres diferentes tasas de crecimiento $\left(r_{A}, r_{B}\right.$ y $\left.r_{C}\right)$. Ello quiere decir que con el MMOM siempre se podrá justificar la recuperación de un volumen de cosecha, aunque éste no sea sostenible. El caso extremo se presenta cuando el volumen de cosecha es $V_{t}$, caso en el cual el MMOM podrá identificar un ciclo de corta que recobre la masa a una tasa exponencial, solución evidentemente imposible. 


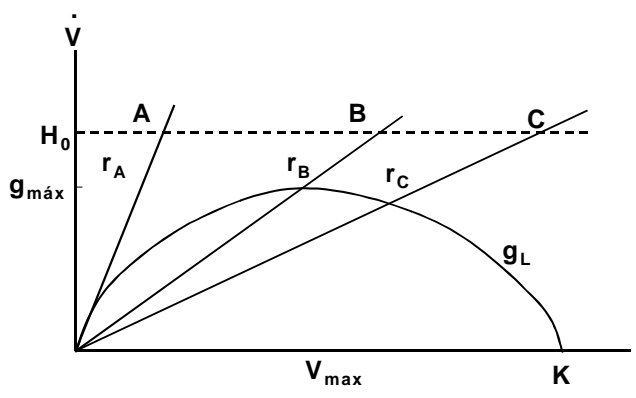

Figura 4. Cosecha superior al máximo crecimiento posible

b) En este caso se supone que el volumen de cosecha es menor al máximo crecimiento posible del bosque, esto es $\mathrm{H}_{1}<$ $\mathrm{t} \mathrm{g}_{\text {máx }} \mathrm{y}$ que la masa sigue un crecimiento logístico (trayectoria gL, Fig. 5). Si además se supone, que una vez realizada la cosecha, el volumen residual es $\left(\mathrm{V}_{0}^{2}\right)$ (Fig. 5), resulta evidente que la masa crecerá a partir de $\left(\mathrm{V}_{0}^{2}\right)$ a una tasa menor que la tasa de cosecha, por lo que se requeriría de un periodo de tiempo $\mathrm{t}+\Delta$ para lograr el volumen de existencias deseado $\mathrm{V}_{\mathrm{t}}$. $\mathrm{Si} \mathrm{H}_{1}$ se sigue conservando de ciclo de corta a ciclo de corta, entonces $\left(\mathrm{V}_{0}^{2}\right)$ será cada vez más pequeña dado que la tasa de crecimiento después de $\left(\mathrm{V}_{0}^{2}\right)$ es menor a la de cosecha. Sin embargo, dado que el crecimiento antes de $\left(\mathrm{V}_{0}^{2}\right)$ es mayor a $\mathrm{H}_{1}$, la masa podrá recuperarse y lograr un equilibrio, poco a poco, muy cercano a $\left(\mathrm{V}_{0}^{2}\right)$.

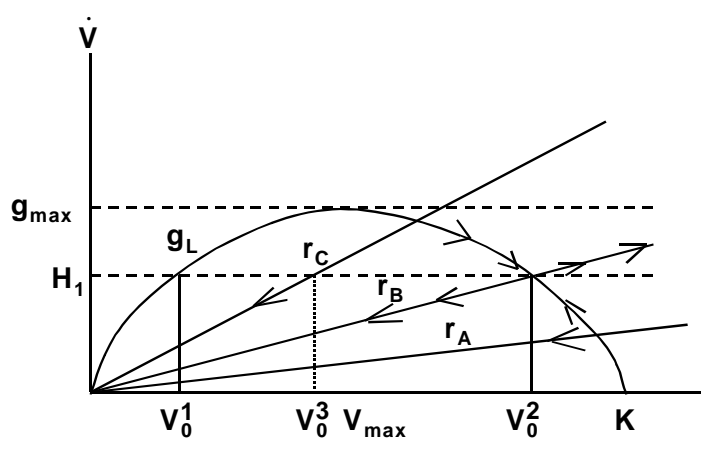

Figura 5. Cosecha sostenible con altas existencias volumétricas.
En este ejemplo $\Delta$ cubre la posibilidad de que la estructura residual de $\left(\mathrm{V}_{0}{ }^{2}\right)$ sea diferente $y$ se obtengan existencias reales diferentes al llegar al periodo t. Por ejemplo, si $\Delta>0$ implicaría que la masa toma más tiempo para lograr las existencias reales iniciales, $V_{t}$, debido posiblemente a que la estructura residual es diferente a la esperada para lograr $V_{t}$ en un periodo t. Otra posibilidad de que $\Delta$ $>0$ es que en lugar de haber dejado como volumen residual $\left(\mathrm{V}_{0}{ }^{2}\right)$ se dejó un volumen residual ligeramente menor, lo que dio por resultado mayor tiempo para $\log r a r \mathrm{~V}_{\mathrm{t}}$.

Si $\Delta>0$ implica que al final del ciclo de corta $t$ el volumen $V_{t}$ es menor al esperado, de aquí que aplicar nuevamente un nivel de cosecha $\mathrm{H}_{1}$ reduciría aún más el volumen residual para el siguiente periodo. Podría pensarse que si $\Delta>0$ se pone en peligro la sustentabilidad del bosque, sin embargo esto no es del todo cierto. Observe en la figura 5 que si el volumen residual es menor a $\left(\mathrm{V}_{0}^{2}\right)$ el crecimiento de la masa es mayor que el nivel de cosecha (definido por $\mathrm{H}_{1}$ ) siempre y cuando el volumen residual sea superior a $\left(\mathrm{V}_{0}{ }^{2}\right)$. De aquí, que aunque se siga aplicando el nivel de cosecha $\mathrm{H}_{1}$, la masa arbolada tendrá la posibilidad de recuperar el volumen $\mathrm{V}_{\mathrm{t}}$; de hecho, sí se mantiene el volumen de cosecha $\mathrm{H}_{1}$ con el mismo ciclo de corta, entonces la población puede llegar al equilibrio de existencias reales sostenibles en el largo plazo $\left(\mathrm{V}_{0}^{2}\right)$.

En estas condiciones se puede establecer que el nivel de cosecha $\mathrm{H}_{1}$ es sostenible en tanto el volumen residual pueda proporcionar una tasa de crecimiento superior a la tasa de cosecha. Evidentemente si $\Delta$ es mayor a cero en cada paso de corta y el nivel de cosecha continúa siendo $\mathrm{H}_{1}$, entonces la masa se está liquidando paulatinamente, sobre todo si $\Delta$ es superior al diferencial de crecimiento entre el crecimiento logístico y el volumen de cosecha. 
Obsérvese que bajo estas condiciones de crecimiento, resulta óptimo conservar el volumen residual en un intervalo alrededor del que proporciona $g_{\text {máx }}$ ya que de esta forma se tiene un mayor crecimiento. Así por ejemplo se puede maximizar el volumen de cosecha $\left(H_{\text {máx }}\right)$ en el periodo $t$ identificando el volumen residual óptimo, esto es:

$$
\mathrm{H}_{\max }=\max _{\mathrm{V}_{0}}\left(\mathrm{~V}_{\mathrm{t}}-\mathrm{V}_{0}\right)=\max _{\mathrm{V}_{0}}\left[\frac{\mathrm{K}}{1+\mathrm{Ce}^{-\mathrm{rt}}}-\mathrm{V}_{0}\right]
$$

La solución a este problema proporciona el siguiente volumen residual óptimo:

$$
V_{0}=\frac{K\left(e^{-\frac{r t}{2}}-e^{-r t}\right)}{1-e^{-r t}}
$$

Ahora considérese que se mantiene un volumen residual $\left(\mathrm{V}_{0}^{2}\right)$ y se calcula el volumen de cosecha con el MMOM. En tales circunstancias se pueden analizar tres escenarios. Si $r$ es muy pequeña (caso más común) el volumen de cosecha $\mathrm{H}_{1}$ (volumen sostenible) es muy alto comparado con el que se calcula con el MMOM ( $r_{A}$, Fig. 5), razón por la cual en el largo plazo el volumen calculado con este método se hará paulatinamente más pequeño, hasta lograr un equilibrio de densidad igual a $\mathrm{K}$ y una cosecha nula. Esta es, sin duda, la razón por la cual la aplicación del MMOM a masas maduras requeriría de coeficientes de ajuste (Rodríguez, 1990) que permitan elevar el valor de $r$. El segundo escenario se presenta cuando $r$ es lo suficientemente grande como para que el volumen de cosecha calculado con el MMOM sea igual a $\mathrm{H}_{1}\left(r_{\mathrm{B}}\right)$. En tal caso obsérvese que la tasa $r$ también es igual a la tasa de crecimiento logística esperada (promedio) en el periodo. Si r permanece constante de ciclo de corta a ciclo de cortan entonces se tendrá un equilibrio de volumen residual que será cercano a $\left(\mathrm{V}_{0}^{2}\right)$ y el MMOM proporciona un nivel de cosecha sostenible.
Sin embargo este caso no sería el esperado dado que $r$ cambia de ciclo de corta a ciclo de corta. Aún más, es fácilmente observable que el equilibrio no es estable, dado que el crecimiento después de $\left(\mathrm{V}_{0}{ }^{2}\right)$ es mayor que el logístico (que implicaría un volumen de cosecha superior) y menor antes de lograr $\left(\mathrm{V}_{0}^{2}\right)$, por lo que si $\mathrm{t}$ $>1$, el equilibrio se perderá y el volumen residual fluctuará alrededor de $\left(\mathrm{V}_{0}^{2}\right)$.

Bajo el tercer escenario $r$ es tan alta que el volumen de cosecha calculado con el MMOM es superior a $\mathrm{H}_{1}$. En tal caso si $r$ permanece constante también existe un equilibrio de largo plazo y es cuando el nivel de existencias residuales es cero. Esto sucede porque un mayor nivel de cosecha representa un volumen residual menor a $\left(\mathrm{V}_{0}^{2}\right)$, mismo que debe proporcionar una tasa $r$ más grande (siguiendo el supuesto del crecimiento logístico). Tal tasa obligaría a un volumen de cosecha más grande y esto finalmente obligará a que la masa se liquide paulatinamente. Lo único que frenaría la extinción es que en algún ciclo de corta se calculara una $r$ inferior a la tasa esperada en el periodo, en tal caso habría un volumen de cosecha inferior que ayudaría a que la masa se recuperara. Esta es sin duda la razón de la aparición de los coeficientes de recuperación aplicados en el MMOM (Rodríguez, 1990), mismos que se utilizan para reducir el valor de $r$ para masas con densidades bajas (que regularmente son menores a $200 \mathrm{~m}^{3} / \mathrm{ha}$ ).

c) En la tercer estrategia de cosecha se presume que el nivel de cosecha sigue siendo $H_{1}$ (p.e. $H_{1}<t g_{\text {máx }}$ ) y que una vez realizada la cosecha las existencias residuales tienen un nivel $\left(\mathrm{V}_{0}^{1}\right)$ como se muestra en la figura 6. En tal caso la tasa de crecimiento futuro es mayor que la tasa de cosecha, por lo que la masa arbolada podrá recobrarse. Repitiendo el ejercicio anterior, considérese que la masa residual es ligeramente menor a $\left(\mathrm{V}_{0}{ }^{1}\right)$, esto es, en el segundo paso de corta será necesaria una cantidad de $\mathrm{t}+\Delta$ años $(\forall \Delta>0)$ para 


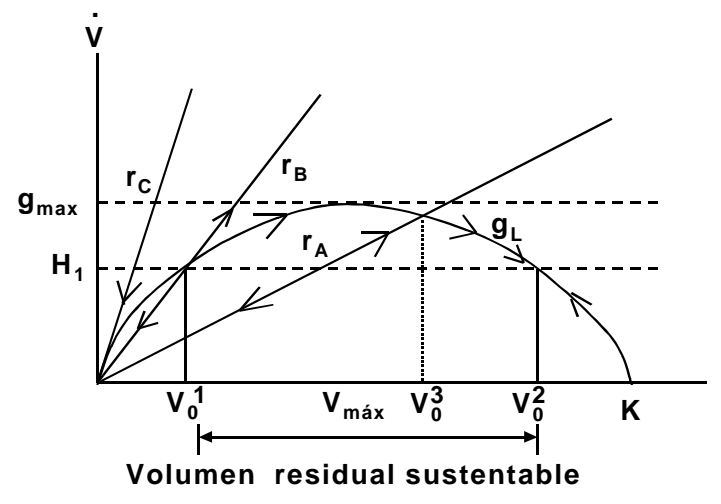

Figura 6. Volumen de cosecha con el mínimo de existencias volumétricas.

lograr las existencias reales iniciales. Obsérvese que en este caso la tasa de crecimiento es menor que la tasa de cosecha, de aquí que si se conserva el nivel de cosecha $\mathrm{H}_{1}$ la masa seguramente se liquidará. Esta condición es sumamente riesgosa, ya que si en los siguientes ciclos de corta se conserva una tasa de cosecha igual a una tasa de crecimiento con el ánimo de recuperar la masa, entonces cualquier desviación de la cosecha que dé por resultado una $\Delta>0$ originará una tendencia hacia la liquidación del bosque. Lo anterior indica que el nivel de cosecha $\mathrm{H}_{1}$ no es sostenible si el volumen residual es igual o menor a $\left(\mathrm{V}_{0}{ }^{1}\right)$. Ello establece que para un nivel de cosecha determinado y manteniendo constante el ciclo de corta, existe un intervalo de volumen residual que es capaz de recuperar la masa y que por lo tanto asegura su sustentabilidad. Este intervalo se ha denominado en la figura 6 como volumen residual sustentable (VRS). Evidentemente, cualquier masa manejada dentro de este intervalo con un volumen de cosecha constante y un mismo ciclo de corta, tenderá en el largo plazo a un equilibrio de volumen residual igual a $\left(\mathrm{V}_{0}^{2}\right)$.

En el caso de calcular el volumen de cosecha con el MMOM existen tres escenarios. En el primero (trayectoria $r_{A}$, Fig. 6) supóngase que $r$ (caso menos frecuente) es inferior a la tasa esperada de crecimiento (trayectoria $g_{L}$, Fig. 6). En tal caso la masa se recuperará y tenderá a un equilibrio de cosecha si $r$ sigue siendo inferior o igual a la tasa de crecimiento esperado en ciclos de corta futuros. Al igual que en el caso anterior el equilibrio es inestable. Si por el contrario la cosecha se mantiene constante en $\mathrm{H}_{1}$ el equilibrio evidentemente es un volumen residual que iguala el volumen de cosecha $\mathrm{H}_{1}$.

En el segundo escenario, $r_{B}$ es similar al crecimiento esperado en el periodo. En tal situación la masa no podrá recuperarse en el ciclo de corta, dado que el crecimiento esperado después de $\left(\mathrm{V}_{0}^{1}\right)$ es inferior al supuesto por el MMOM. De aquí que las existencias residuales sean menores que $\left(V_{0}^{1}\right)$. Si $r$ se mantiene y $V_{t}$ es menor que $\left(\mathrm{V}_{0}^{1}\right)$ la masa puede recuperarse, de lo contrario no hay recuperación. En este caso, en el que las tasas son más grandes se puede apreciar que un ciclo de corta más largo tiene más riesgo de una sobre-explotación. Finalmente, si $r$ es superior al crecimiento esperado en el periodo, el volumen de cosecha calculado con el MMOM será siempre superior al volumen que se podrá recobrar, por lo que la masa se liquidará paulatinamente, a menos que en los ciclos de corta futuros $r$ sea menor al crecimiento esperado en el periodo.

d) En la cuarta estrategia, suponga que el nivel de cosecha es $\mathrm{H}_{2}$ y equivalente al máximo crecimiento de la masa $\left(\mathrm{H}_{2}=\mathrm{t}\right.$ $\left.g_{\text {máx }}\right)$. En este caso, presumiendo un crecimiento logístico, el nivel de cosecha sólo es sostenible si $t=1$, ya que si $t$ es mayor a la unidad, entonces el volumen de cosecha será superior al crecimiento de la masa, y ésta tenderá a liquidarse. De aquí que el nivel de cosecha $\mathrm{H}_{2}$ no es sostenible en la práctica. Si el nivel de cosecha se calcula con el MMOM y el volumen es similar a $\mathrm{H}_{2}$, evidentemente el nivel no es sostenible, dado que forzosamente el MMOM estimará un 
volumen residual diferente a aquel que brinda el máximo crecimiento.

\section{RESULTADOS}

Con base en el análisis desarrollado en la sección anterior se pueden derivar algunos resultados del método de regulación de la corta por volumen de cosecha, calculado ya sea con una función de crecimiento de comportamiento logístico y aquel volumen de cosecha calculado con una función de crecimiento exponencial. Considerando la función de crecimiento logístico se pueden rescatar los siguientes resultados:

a) Un volumen de cosecha igual 0 superior al máximo crecimiento en volumen no es sostenible.

b) Existe un rango de densidad de existencias residuales (Volumen Residual Sustentable) que asegura la sostenibilidad de un nivel de cosecha y un ciclo de corta fijo. La figura 6 muestra que una tasa de cosecha $\mathrm{H}$ menor al máximo crecimiento puede ser sostenible indefinidamente si el volumen residual es mayor a K/2, o bien, es mayor o igual al mínimo volumen que puede generar una tasa de crecimiento igual a la tasa de cosecha. Esto es, sí:

$$
\dot{V}=\frac{d V}{d t}=H
$$

y

$$
V_{0}(V)\left\{\begin{array}{l}
V>\frac{K}{2} \\
\frac{d V}{d t}>H \quad \forall V<\frac{K}{2}
\end{array}\right.
$$

c) $\mathrm{Si}$ el volumen de cosecha $\mathrm{H}$ es sostenible y se cumplen los requisitos del punto anterior, entonces la masa tiende a un equilibrio (estable) y el nivel de cosecha se puede regular, independientemente de las variaciones en crecimiento que se pueden derivar de tener estructuras residuales diferentes.

d) Si la masa tiene una función de crecimiento cóncava es posible determinar el volumen de existencias residuales que optimice el crecimiento en un ciclo de corta t. El modelo (6) estima el volumen residual que optimiza el crecimiento suponiendo una función de crecimiento logística. Este modelo sólo confirma los siguientes resultados ya conocidos del método de regulación por volumen:

i Si la tasa de crecimiento exponencial $r$ es mayor (lo cual se puede lograr con sitios de mejor calidad para una especie), entonces el volumen residual $\left(\mathrm{V}_{0}\right)$ óptimo puede ser menor ceteris paribus.

ii Si se incrementa el ciclo de corta $t$ (ceteris paribus) entonces el volumen residual $\left(\mathrm{V}_{0}\right)$ óptimo puede ser menor.

iii Si la especie es más tolerante a la sombra, entonces la capacidad de carga $(\mathrm{K})$ puede ser mayor. En tal caso el volumen residual $\left(\mathrm{V}_{0}\right)$ óptimo debe ser mayor ceteris paribus.

iv En la medida en que el ciclo de corta se hace más pequeño (p.e. $t \rightarrow 0$ ) el volumen residual óptimo tiende a ser el que proporciona el máximo crecimiento, esto es K/2. Por el contrario, a medida que el ciclo de corta se alarga (p.e. $t \rightarrow \infty$ ) el volumen residual óptimo tiende a cero. Lo anterior indica que el volumen residual óptimo está en el intervalo $0 \rightarrow \mathrm{K} / 2$, criterio que ayuda a explicar que en una masa virgen pueda resultar óptimo cosechar el $50 \%$ o más de la masa, tal como aquellas cortas realizadas en Atenquique, Jalisco entre 1955 1965 (DGAF, 1984b). Obviamente en 
tales casos es necesario readecuar los volúmenes de cosecha $y$ volúmenes residuales para los siguientes ciclos de corta.

e) Si el volumen residual es menor al mínimo volumen que puede generar una tasa de crecimiento igual a la tasa de cosecha $\left(\mathrm{V}_{0}{ }^{1}\right.$ en la figura 6$)$, entonces la cosecha no es sostenible y se tenderá a liquidar el bosque.

f) En la medida que el nivel de cosecha $\mathrm{H}$ se aproxima al máximo crecimiento en volumen entonces se acorta el rango del volumen residual sustentable (VRS) hasta llegar a su mínimo nivel de holgura cuando estas existencias equivalgan al volumen que proporciona el máximo crecimiento ( $\left.g_{\text {máx }}\right)$.

g) Si el volumen de cosecha y el ciclo de corta son fijos, y si además este volumen de cosecha cumple los requisitos en b) se tenderá a la regulación de la cosecha, independientemente de la estructura de la masa residual.

Estos resultados sólo indican que una estrategia de regulación por volumen puede sostener un volumen de cosecha, teóricamente en forma indefinida, independientemente de la estructura residual. Ello es factible en el caso de que el modelo de crecimiento suponga rendimientos marginales decrecientes. Evidentemente se debe trabajar en el conocimiento de las características más apropiadas de la estructura residual para que permitan mantener productividad, salud, biodiversidad y producción de múltiples bienes y servicios a fin de que se pueda lograr una estructura residual sustentable.

Si por el contrario se presume que la masa crece a una tasa constante, entonces se pueden rescatar los siguientes resultados. En ellos se supone que la forma de crecimiento exponencial representa el crecimiento esperado de la masa.

a) Cualquier volumen de cosecha calculado con el MMOM puede ser justificable con sólo variar el ciclo de corta, independientemente de que tal volumen sea sostenible o no.

b) El volumen de cosecha calculado con el MMOM es sostenible si $r$ es igual o menor a la tasa esperada de crecimiento en el periodo (ciclo de corta) y si el volumen de cosecha es menor al máximo crecimiento esperado que se pueda lograr en cualquier periodo.

c) Dado que $r$ es dependiente de la densidad y edad de la masa y considerando que a menor densidad $o$ edad se espera una mayor $r$, es muy probable que en masas jóvenes exista mayor peligro de liquidación que en masas maduras 0 con mayores densidades.

d) El MMOM puede estimar volúmenes de cosecha casi constantes, siempre y cuando la estimación de crecimiento (exponencial) en el periodo coincida con el crecimiento esperado en el mismo periodo y que estos crecimientos (netos) sean equivalentes al volumen de cosecha. Sin embargo, no es posible asegurar que una repetida aplicación del MMOM estime volúmenes de cosecha sostenibles.

e) Mientras más largo sea el ciclo de corta se corre mayor riesgo de que el crecimiento exponencial refleje volúmenes que no se podrán recobrar.

f) Diferencias en $r$ definidas por el MMOM reflejan diferencias en tasas de crecimiento de volúmenes residuales, mas no reflejan diferencias en calidad de sitio como sería el caso de la función de crecimiento logística. 
g) El supuesto de crecimiento exponencial del MMOM, no permite calcular una densidad residual óptima, dado que para este procedimiento siempre será óptimo cortar lo más posible y alargar el ciclo de corta. Esto es resultado de que mientras mayor sea el ciclo de corta mayor será la acumulación de crecimiento exponencial. Además, tal y como lo muestra el diagrama de fase de la figura 6 , los únicos equilibrios son la liquidación de la masa o la capacidad de carga. Este resultado sólo muestra que no es posible regular el volumen de cosecha siguiendo el procedimiento de cálculo del MMOM. De aquí que para mantener las existencias en un nivel apropiado siempre será necesario emplear ya sea coeficientes de recuperación o bien coeficientes de ajuste.

Lo anterior indica que la limitante más importante del MMOM es el supuesto de crecimiento exponencial, mismo que imposibilita a este método a regular el volumen de cosecha en el largo plazo. Sin este requisito el MMOM no puede garantizar la sostenibilidad de un volumen de cosecha. Si a esta limitante se agrega que no existe ni una estrategia de manejo de estructuras residuales, ni una concepción de regulación de cosecha a nivel paisaje asociada al método, entonces el MMOM no es un procedimiento que asegure la sustentabilidad del bosque.

Los principios del MMOM pueden aplicarse a un modelo de crecimiento logístico, en el cual existan criterios de comparación de volúmenes de cosecha, exista la clara definición de una población residual sustentable y que sea posible identificar estrategias eficientes de manejo forestal. Ello anterior requiere de una evaluación de la función de crecimiento (bajo el supuesto de rendimientos marginales decrecientes) con parámetros como la capacidad de carga (K), o la tasa exponencial de crecimiento ( $r$ ). Tales parámetros son variables de especie a especie y de sitio a sitio, de aquí que la aplicación de estos criterios a casos reales podría no resultar del todo simple. El modelo logístico puede simplificar enormemente la estimación, ya que $\mathrm{K}$ puede estimarse a través de guías de densidad o simples datos de inventario, mientras que $r$ se puede estimar a partir de datos de incremento. Así por ejemplo, si se cuenta con una estimación de $\mathrm{K}$ entonces es posible estimar $r$ a partir de una estimación de crecimiento en volumen con la expresión:

donde:

$$
r=\frac{\log \left(\frac{c}{c^{\prime}}\right)}{t}
$$

$$
c=\frac{K-V_{0}}{V_{0}} \quad y \quad c^{\prime}=\frac{K-V_{t}}{V_{t}}
$$

Estos resultados establecen que es complicado poder evaluar la sostenibilidad del volumen de corta estimado con el MMOM si no se cuenta con criterios de comparación, esto es, si no se sabe cuál es la capacidad de carga (K), cuál es el crecimiento esperado en el periodo, cuál es el máximo crecimiento (periódico) posible y si los rendimientos marginales son crecientes o decrecientes. Si estos elementos están disponibles a través de estimaciones simples de crecimiento con un comportamiento logístico entonces es posible identificar criterios de evaluación de los niveles de cosecha y definir si tales niveles son sostenibles o no. Así por ejemplo, la evaluación del volumen de cosecha (posibilidad) debe reconocer que:

A) El volumen de cosecha debe compararse con el máximo crecimiento periódico posible de la especie o de la mezcla de especies y para la misma calidad de sitio. Dicho volumen debe ser estrictamente menor que el máximo crecimiento periódico posible. 
B) Dado que el mismo nivel de cosecha se logra dentro de un amplio rango de densidades, es conveniente asegurar que la densidad residual sea superior a $\mathrm{K} / 2$. Lo anterior implica que la densidad residual sea superior a aquella densidad que proporciona el máximo crecimiento periódico posible.

C) La liquidación de un bosque excedente (p.e. un bosque con volumen cercano a su capacidad de carga $K$ ) puede realizarse reduciendo la densidad a un mínimo de K/2.

D) Si la densidad residual es menor a aquella densidad que proporciona el máximo crecimiento corriente anual será indispensable identificar la mínima densidad que proporciona un crecimiento periódico similar al nivel de cosecha. Obviamente, la densidad residual deberá ser mayor a esta densidad mínima.

E) Si la densidad residual es menor a la mínima densidad que proporciona un crecimiento periódico similar al nivel de cosecha no debe reducirse el nivel de cosecha para igualar el crecimiento periódico, dado que los resultados son inciertos y dependientes de la distribución residual. $\mathrm{Si}$ esta distribución es de arbolado joven es probable que se iguale o supere el volumen de cosecha, de lo contrario se tenderá a una liquidación paulatina de la masa.

F) Mientras más cercano sea el volumen de cosecha al máximo volumen de crecimiento periódico, el volumen residual mínimo aceptable debe ser más cercano a K/2, esto es, a aquel volumen residual que proporciona el máximo crecimiento periódico posible.

Estos criterios de evaluación de un volumen de cosecha sólo garantizan que tal volumen sea sostenible a través del tiempo y que no se liquide la masa, sin embargo no garantizan algún tipo de eficiencia económica. En este sentido, si se supone un crecimiento logístico es posible identificar una estrategia de cosecha económicamente eficiente y sustentable, sin embargo, si se supone un crecimiento exponencial es prácticamente imposible encontrar una solución de manejo económicamente eficiente.

\section{CONCLUSIONES}

En el presente trabajo se ha mostrado que la debilidad más grande del MMOM es el supuesto de crecimiento exponencial durante el ciclo de corta. Ello hace que el MMOM pueda calcular un nivel cosecha sostenible sólo en condiciones muy particulares. Incluso dadas tales condiciones es muy probable que no se vuelvan a repetir (el equilibrio no es estable) en ciclos de corta sucesivos. Adicionalmente se ha mostrado que es prácticamente imposible definir indicadores de sostenibilidad del volumen de cosecha bajo el supuesto de crecimiento exponencial y si se ignoran parámetros de la población tales como máximo rendimiento periódico posible, capacidad de carga y volumen residual sustentable. Lo anterior establece que si la dasonomía mexicana desea adoptar criterios de sustentabilidad en el manejo forestal es imperativo modificar los supuestos del MMOM, incluir indicadores de sotenibilidad e la cosecha, monitorear los resultados obtenidos en el largo plazo y definir criterios de manejo de estructuras residuales que permitan mantener la biodiversidad, salud, productividad, así como producción de múltiples bienes y servicios en el largo plazo.

\section{REFERENCIAS}

Assmann, E. 1970. The principles of forest yield study. Pergamon Press. Nueva York. 506 p. 
Adams, D.M. y A.R. Ek. 1974. Optimizing the management of uneven-aged forest stands. Can. J. For. Res. 4:274.287.

Clark, C. 1990. Mathematical bioeconomics: The optimal management of renewable resources. $2^{\text {nd }} \mathrm{Ed}$. Wiley and Sons. 306 p.

Davis, K.P. 1956. Determination of desirable growing stock, a central problem of forest management. J. For. 54:811-815.

Dirección General de Aprovechamiento Forestales. 1984a. Normas mínimas de calidad para la formulación de estudios dasonómicos en bosques. Subsecretaría Forestal. SARH. 9 p.

Dirección General de Aprovechamiento Forestales. 1984b. Antecedentes y evolución del Método Mexicano de Ordenación de Bosques Irregulares. Subsecretaría Forestal, SARH. 13 p.

Guldin, J.M. 1991. Uneven-aged BDq regulation of Sierra Nevada mixed conifers. Western J. Applied For. 6: 27-32.

Haight, R.G., J.D. Brodie y D.M. Adams. 1985. Optimizing the sequence of diameter distributions and selection harvests for uneven-aged stands management. For. Sci. 31: 451.462.

Klepac, D. 1976. Crecimiento e incremento de árboles y masas forestales. Dpto. de Bosques. UACH. Chapingo, México. 365 p.

Lagsaeter, A. 1941. Omitynning i analdret granfurvskog Maddel. Norsite Skogfor soksvenson 8:131-216 (citado por Smith, 1986).

Leak, W.B. y S.M. Filip. 1977. Thirty eight years of group selection in New England northern hardwoods. J. For. 75:641-643.
Long, J.N. 1998. Multiaged systems in the central and southern Rockies. J. For. 96:34-36.

Mar-Moller, C. 1960. The influence of pruning on the growth of conifers. Forestry 33:37-53.

Mendoza M., R. y R. Rodríguez C. 1959. Método mexicano de ordenación de montes. Apéndice $7 \mathrm{del}$ Proyecto Gral. de Ordenación Forestal de la UIEF Michoacana de Occidente. Unidad Industrial de Explotación Forestal Michoacana de Occidente, S. de R.L. Uruapan, Mich. 82 p.

Oliver, C.D. y B.C. Larson. 1990. Forest stand dynamics. McGraw Hill. 470 p.

O'Hara, K.L. 1996. Dynamics and stocking level relationships of multi-aged ponderosa pine stands. For. Sci. Monograph No. 33.

O'Hara, K.L. 1998. Silviculture for structural diversity: A new look at multiaged systems. J. For. 96:4-10.

Reynolds, R.R., J.B. Baker y T.T. Ku. 1984. Four decades of selection management on the Crossett Farm Forestry Forties. Bull 872. Fayetteville, Arkansas. Agric. Exp. Stat. 87 p.

Rodríguez, C.R. 1958. Discusión de fórmulas para el cálculo de la productividad maderable y exposición del Método Mexicano de Ordenación de Montes de especies coníferas. Monografía Ftal. del Edo. de Michoacán. Comisión Ftal. del Estado. 245 p.

Rodríguez, C.R. 1990. Segundo Estudio Dasonómico del Estado de México: SEDEMEX: Gob. Edo. de México Sec. Desarrollo Agropecuario. Protectora de Bosques. 334 p. 
Rodríguez C., R. y M. Rodríguez C. 1966. Generalización del Método Mexicano de Ordenación de Bosques. México y sus Bosques. Sep-Dic:2-4.

Seymour, R. y L.S. Kenefic. 1998. Balance and sustainability in multiaged stands: A Northern conifer case study. J. For. 96:12-17.

Smith, D.M. 1986. The Practice of Silviculture. $8^{\text {th }} \mathrm{Ed}$. Wiley and Sons. Nueva York. 527 p. 